\title{
Insect Detection in Rice Crop using Google Code Lab
}

\author{
K. Sumathi ${ }^{\mathrm{a}}$, G. Depshikha ${ }^{\mathrm{b}}$, M. Dhivya ${ }^{\mathrm{c}}$, P. Karthika ${ }^{\mathrm{d}}$ and B. Priyanka ${ }^{\mathrm{e}}$ \\ Department of Computer Science and Engineering, M.Kumarasamy College of \\ Engineering, Karur, Tamil Nadu, India -639113 \\ b,c,d,e Department of Computer Science and Engineering, M.Kumarasamy College of Engineering, Karur, Tamil Nadu, India - \\ 639113
}

Article History: Received: 11 January 2021; Accepted: 27 February 2021; Published online: 5 April 2021

\begin{abstract}
Herb plants are essential in the medical field today and can help humans. Phyllanthus Elegans Wall is used in this study to analyse and categorize whether it is a safe or unhealthy leaf. At the moment, most insect identification methods rely on physical classification, making it difficult to automatically, quickly, and reliably identify in stored grains. The concept of this research is to ascertain the quality of leaves by combining technology with pesticide classification in the agricultural sector. Picture collection, image processing, and classification are the first steps in enhancing leaf quality analysis. The segmentation using HSV to input RGB image for the colour alteration structure is the most significant image processing method for this section. The colour and shape of a leaf disease image are used to analyse it. Insect detection in complex backgrounds is more versatile with the score map that is decision alternate highly interconnected layer, and our detection speed has upgraded. Finally, the taxonomy approach employs an algorithm that feeds directly that employs formation backwards techniques. The result shows a Many-layer Preceptor and Nonlinear Activation Feature comparison, as well as a percentage of accuracy contrast between MLP and RBF. MLP and RBF are neural network algorithms. Clearly, the Neural Network classifier has a better presentation and precision.
\end{abstract}

Keywords: Quality of Leaf, Image Acquisition, Image Processing, Stored-grain Insect, RBF and Multi - layer perceptron (MLP).

\section{Introduction}

Its aim is to build a capability in the cultivation field through engineering collaboration. Crops are now subjected to a variety of traits and diseases. One of the most primary causes of post-harvest losses are insect [1], with the help of system which is used to monitoring the insects in the stored grain are detected. The most important traits/diseases are insect-caused foundation damage. Insecticides are not necessarily well-organized, as certain insecticides are harmful to some types of birds. It also has an adverse effect on natural animal food chains. Plant scientists' standard practice is to measure plant damage (e.g., leaf, branch) based on the quantity of diseased area observed by naked eye on a broad scale. This paper introduces an innovative approach for researching plant diseases and traits using image processing.

Based on computer visualization the outcome of the insect texture, form, and local independence has long been a subject of in the research field of insect classification. [11] built an insect detection system with a MAP (mean average precision) of greater than 75 percent on 24 common insects in grain production, researchers used a coding that is sparse for programming shots of insects and system methodology. Lim et al [12] give permission to Alex net and Soft Max to develop an insect arrangement framework that was optimized using network architecture regulation. Using four-feature extraction, the image-based insect classification system was proposed by Yalcin [13].

The scheme under consideration is for increasing throughput while reducing error caused by human experts being aware of plant diseases. Leaf disease identification and analysis using engineering technologies and mathematical theory in Artificial Neural Network (ANN). In comparison to most conventional classification approaches, ANN has been used in agriculture image processing frequently and it is the most popular methods for ordered. The Neural Network will identify the sample data of leaf representation based on whether it is safe or unhealthy at the end of this learning. The leaf image from an aromatic plant was used in this creation. Using an Artificial Neural Network, the image acquisition system uses appropriate image processing algorithms to classify and differentiate healthy and unhealthy leaves. This project's image tagging prototype is based on leaf shade and area. This growth is linked to the cultivation sector's production strategy. Leaf Syndrome Classification Using Artificial Neural Network is the title of this project. Extort the image by using an appropriate image processing algorithm. Then, using an Artificial Neural Network, assemble awareness and organization of safe or unhealthy leaves. This development's image sorting pattern is focused on the colour and area of the leaf.

\section{Literature Review}

[1] India is an agricultural nation, with agriculture providing employment to the majority of the population. This approach is opulent since it necessitates professional supervision at all times. As a result of the use of technology in agriculture, this scheme can be used for a long time and at a lower cost. Plant diseases have become a concern because they can result in substantial reductions in both the quality and quantity of undeveloped goods. [3] Crop farming is one of the most important aspects of the country's economy. The superiority and quantity of 
undeveloped output are influenced by environmental factors such as rain, temperature, and other weather conditions that are beyond human control. Another major constraint to crop efficiency is pathogens, which humans may coordinate to improve both quality and quantity efficiency. Farmers in rural areas have limited access to undeveloped experts who can examine crop images and provide advice, and they have a wide variety of crops to choose from. [4] The crop cultivation for maximum and superior production, on the other hand, is a highly technological method. It can be improved with the assistance of information support. The exposed eye approach is commonly used to diagnose diseases. In this method, only those with the ability to see changes in leaf color are involved. This approach necessitates a great deal of work, takes a long time, and is unsuitable for large fields. Often, different experts will diagnose the same disease as the same disease.

[5] IoT is an open-source project that aims to connect things like Smartphone's. Sensors and their messages are controlled by back ends, an application that provides an Application programming interface wants to access the data. Using Grid Cloud test bed, the platform is demonstrated with video sensors. The program can be downloaded from GitHub. Another open-source initiative spawned by an EU-funded research project is Open IoT. [6] An introduction of computing in the fog system for agriculture precision. The couple-leveled structure decreases the amount of data sent through the network substantially. A key advantage of the fog approach is that it reduces tonnes of computation, more importantly; the cost of the cloud storage is associated with centralized functioning. [7] The authors suggest a hybrid Internet of Things (IoT) system for digital agriculture in remote region for field interfaces, the contact service employs 6LoWPAN local radio, and distanced networks are made. To ensure cross-domain convergence of the farm range connection, transitional distanced switches, and stored data, a 6LoWPAN boundary switches and a specialized portal. Throughput, latency, and accessibility support are all addressed in terms of network specifications for smart agriculture applications. This provides a useful benchmark for measuring the grouped information capacity in relation to needs of monitoring.

[8] Introduces a segregated computing design that includes basic agricultural system mechanisms are seed, sand, atmosphere, and electricity. [9] To connect monitors, modelers, contact network, computers, and the messaging mechanism is standardized all over the sensor data. For in situ decision-making, a tree is constructed for watering and coordinated on service provider. Cloud platforms provide data into an edge customer interface with top-range evaluation.[10] Presents a circulated sensor-driven clever water system framework dependent on wide range, minimum hubs and portal. FIWARE transportation stage, which offers support admin, is utilized as information processing middleware stage. Multiple operation scenarios are tended to as far as a many hub, based on scalability criteria. [11] IoT monitoring on a large scale, that emphasis ground-level grouping mechanisms that helps to collect information and to check event generation. Support for aerial robotic platforms is provided by sufficient high-level trajectory control for data collection and backhaul. [12] Thresholding is local computed moving minimum in combination which accomplished information modified is reduced. To achieve reliable Transmissions, A Variety Of Radio Access Technologies Are Available.

\section{Methodology}

\subsection{Image Processing Method}

Centered on S. Arivazhagan et al. [2], a system for identifying and classifying leaf diseases has been proposed. Masking and removing green pixels, applying a special doorstep to remove the infected area, and computing texture statistics to assess the disease are all part of the segmentation process. Since it is based on human interpretation, his color model (hue, saturation, intensity) is a common color model. The color co- occurrence function is used to calculate consistency. According to their findings, co-occurrence is a mathematical way of describing form by statistically demonstrating how such gray-levels occur in close proximity to other levels. Based on their research, they classified plant diseases into different groups. New Jersey JanweandVinitaTajane [12] suggested using the Canny Edge detection algorithm, Histogram psychoanalysis, and CBIR for disease detection in their medical plants. Identification of medicinal plants based on their edge characteristics. Calculate the edge histogram by converting the leaf image to grayscale. The algorithm that was developed for this purpose is called canny edge discovery. The histogram for the overall image is used to extract the image's neighborhood and shadow. Based on their study, they discovered that the color histogramcanbeusedtodistinguishthelayersofanRGBillustrationintored, green, and blue color histograms in order to verify the strength of each color pixel in a difficult situation, which is useful for recognizing color.

\subsection{CNN Feature Extraction Network}

Convolutional Neural Networks (CNNs) are a type of neural network that is stimulated by the biological visualization cognitive mechanism. The convolution operation is located in the middle of the Convolutional neural network. As a consequence, in image recognition, object detection, and other tasks, a Convolutional neural network outperforms other computer vision algorithms. Without complex image preprocessing, a Convolutional neural network may extract the successful features from a large number of original input data, making image feature extraction simple and unbeatable.

\subsection{Classification}

Five data improvement approaches were believed to extend the training set in order to enhance the training set and improve the model's simplification the prospects. The following are the models: 1) The insect's unusual postures through horizontal mirror image; 2) To boost the model and Gaussian noise to the training collection .The image brightness adjustment model will detect insects in scenes with varying brightness level; 4) Rotated images to establish insect orientations and postures; 5) By extracting different sections of the original image ,the 
training set may provide more images with various panorama .Our latest data volume is 10,296 images, are nearly 144,000 insect images ,thanks to these methods

Many articles have been published in international journals and conferences, and researchers have focused on approaches such as hierarchical, neural networks, and machine learning medium. The classification of leaves began in the early twentieth century [13].Support Vector Machine (SVM), neural network, conventional manifold regression, k-Nearest Neighbor, and Genetic Algorithm are some of the classifier techniques that pass researchers frequently used. In [14], Prasad Babu et al proposed a Back proliferation neural network for leaf acceptance. It was demonstrated that a back transmission network and a silhouette of a leaf picture are sufficient to identify a leaf's species. Tzionas et al developed a computer vision method that eliminates explicit geometrical and morphological features [13]. A subset of significant image skin tone was recognized using an over feature assortment detain. Back Propagation Method A multi-layer preceptor is trained using the back-propagation algorithm, which is a methodical technique (MLP). Back propagation is a problem-solving algorithm that can be applied to a variety of situations. There are three phases to the back-propagation analysis algorithm:

I. Back-propagation of the connected mistake

II. Feed-forward of the participation planning pattern

III. Altering your weight

Amultilayernetcanonlylearntheinputpatterntoanimpreciselevelofaccuracy.Amultilayer feed forward neural with an input layer, production layer, and secret layer is known as an MLP. The prejudices of the neurons in the hidden and output layers are comparable to weights on relationships from units whose output is always 1 [15]. The commonly delta law was introduced, which resulted in the back-propagation algorithm. It's a gradient descendent approach that reduces the total squared error of a collection of connections' performances [15]

\subsubsection{Feed Forward the Input Training Pattern}

Every input neuron (Xi) gets an input data indication and broadcasts it into every unrevealed neuron, which computes the commencement and passes it on to each output value node, which then calculates the origination to receive the total output.

\subsubsection{Back Propagation of Associated Error}

The net performance is checked to the target value during the planning and the associated mistake is taken into account. The error factor $\delta \mathrm{k}$ is calculated from the error and helps to obtain the error to the layer of hidden.

\subsubsection{Weight Adjustment}

When an error occurs, the heaviness must update to separate the error back into the unrevealed layer. The error factor $\delta \mathrm{j}$ for unit $\mathrm{Z}$ j is determined using a similar process. The weights are changed at the same time as the error problem is resolved.

\section{Model Optimization}

By building on the original DenseNet-121, we make use of the latest DenseNet-121 in this project as the extraction of networks in CNN features. Due to the original Densenet-121, we replaced the 3 X 3 transform in the 4th Dense Block with a wide method is structured. We compressed the parameters and made the network lighter in this way, and accuracy improved as a result.

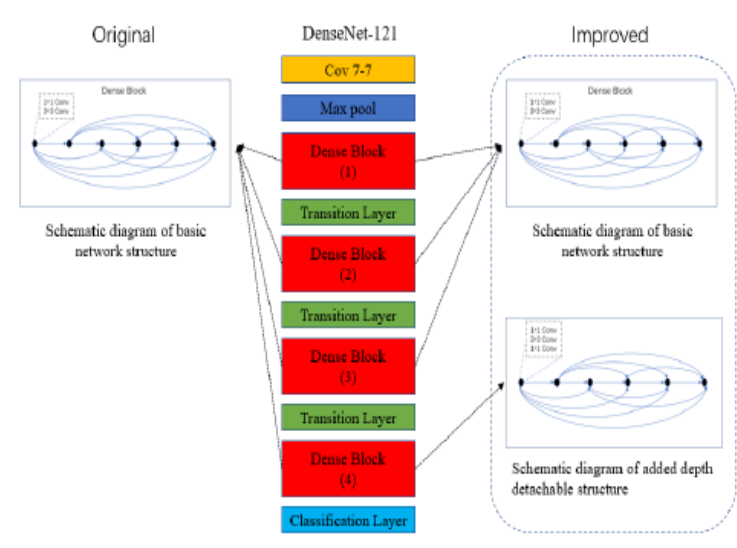

Figure 1: Advanced DenseNet-121 Structure

The initial structure is the original part; the enhanced part is that we configure the network through deep separable convolution. This describes the steps involved in determining the classification of leaf disease. The first step is to obtain a picture using an elegant phone with an 8-megapixel sensor. The image allowance system is used to take fifty samples of vigorous leaf images and fifty samples of unhealthy leaf images Contrast enhancement, segmentation, and feature extraction are the three mechanisms that make up the image processing process. Finally, using an Artificial Neural Network, the compiled data would be confidential to the health or unhealthiness of leaves. 


\subsection{Classification Technique}

Using the Back-propagation algorithm, the corporeal situation of the leaf is kept private in this process. The back-propagation algorithm is a method for training a multilayer preceptor that is methodical (MLP). (RBF) will be compared to (MLP) to get good accuracy to result in terms of arrangement precision. A health categorization of leaves is done using java in this process. This is an example of splitting the leaf's hidden data into two sections. This information was gathered from data image dispensation based on colour and shape to assess categorization management.

\subsection{Performance of MLP}

In categorization problems modelled by neural networks, there are three distinct phases: network construction, learning, and testing. There are three layers in a back transmission network: a two-parameter input layer, an unrevealed layer with ten neurons, and an output layer that surrounds a single neuron. In the learning section, the weights of the machine relation are set haphazardly. Between 0 and 1, the input parameters are normalized. The mean of squared errors is used to determine the network's shape.

\subsection{Performance of RBF Network}

The same debate took place at the presentation network, which was used to train and test the RBF framework. This is the same practice saving an MLP presentation. Both the training sample and the test sample were given the identical worth. When the sum of testing some explanations exceeds a sum of test samples, 99.2 percent of the leaves are correctly labelled. There is a 0.8 percent margin of error.

\section{Result and Discussion}

The weight variables of the changed layer were initialized using the MSRA initialization process and qualified Dense Net weight variables. The basic frame was created using the Image Net data collection.

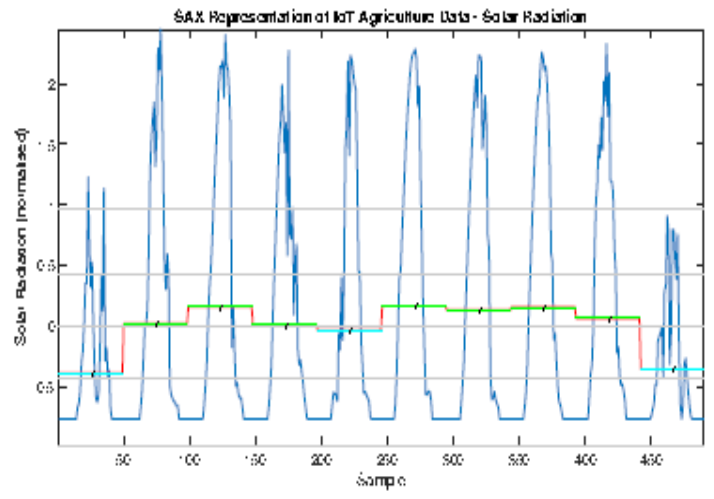

Figure 2: Symbolic Aggregation

The gradient descent that is adaptable Adam was a computer programmer used to get it up to date back propagation variables during the testing, as well as the extended training set revised and It was repeated. The algorithm's failure curve is shown in FIGURE 4.

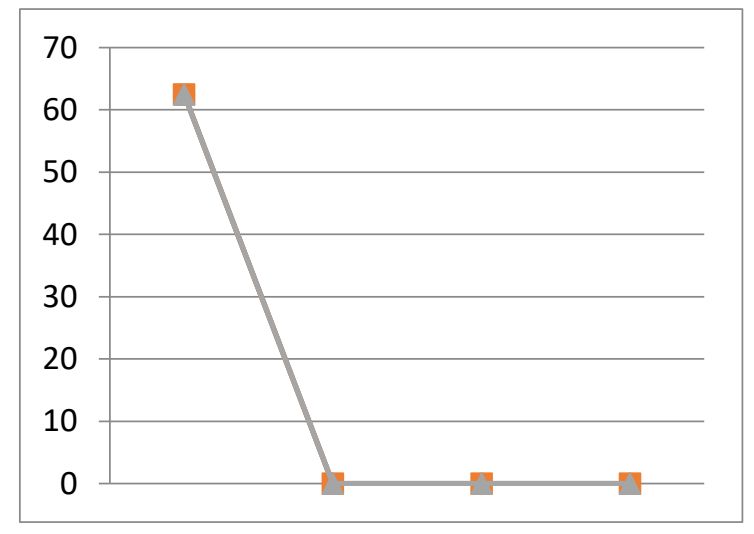

Figure 3: Shows the loss graph above 60k iterations.

Enhancement of data may enhance the data set's abundance, allowing The CNN is a form of neural framework that uses to have a better understanding of the functionality that are more generalized and consequently boost recognition. In Table.2, the overall precision of the data improvement R-CNN is faster which improves around 4 percent. From its inception, results had been clear and the idea had been sound. The use of Res Net- 10 increased overall performance by around 3percent as compare the two CNN under the Faster R-CNN algorithm, the two CNN the framework might be able to get improved picture capabilities and thus enhance the detection algorithm's output. The framework identification speed was significantly enhanced by increasing the mutual complexity 
service and decreasing the cost duplication measurement, so the possibility that recognition a single's tempo picture had been decreased with an average of $0.217 \mathrm{~s}$ to $0.124 \mathrm{~s}$ precisionof 83 percent when contrasting R-FCN and Faster R-CNN are two types of R-FCN. R-recognition FCN's effect was greatly increased by the enhanced image recognition system.

\section{Conclusion}

The performance of the data has been successfully measured using image recognition system and secret using Neural Framework to get the performance of the data. The image allowance system was used on 100 leaf samples, with data dependent on colour and unhealthful area studied. Using an image processing tool, the aim of capturing and analysing data from leaf images in order to arrange robust or unhealthy leaves of drug plants was accomplished. To retrieve the image and obtain data, an algorithm of attuned contrast, segmentation, and features removal is used from the image processing system. The image processing approach incorporates these three methods. Artificial Neural Networks were used to simulate the experimentation results. The Architectures of the framework second-hand to class stable or dysfunctional leaves are multi-layer feed forward several layer and radial base function $\mathrm{RBF}$.

\section{References}

1. K. Patil. Jayamala, and Raj Kumar. 2011. Color Feature Extraction of Tomato Leaf Diseases. International Journal of Engineering Trends and Technology. 21(2): 72-74.

2. Arivazhagan, S. et al. 2013. Detection Of Unhealthy Region Of Plant Leaves And Classification Of Plant Leaf Diseases Using Texture Features. CIGR Journal. 15(1): 211- 217.

3. Murugesan, M., Thilagamani, S. ," Efficient anomaly detection in surveillance videos based on multi layer perception recurrent neural network", Journal of Microprocessors and Microsystems, Volume 79, Issue November 2020, https://doi.org/10.1016/j.micpro.2020.103303

4. N. Ghaiwat, Savita, and ParulArora. 2014. Detection and Classification of Plant Leaf Diseases Using Image Processing Techniques: A Review. Department of Electronics and Telecommunication Engineering, Wagholi, Pune. 2(3): 1-7.

5. Thilagamani, S., Nandhakumar, C. ." Implementing green revolution for organic plant forming using KNNclassification technique”, International Journal of Advanced Science and Technology, Volume 29 , Isuue 7S, pp. 1707-1712

6. Saad, Hashim. 2015. Detail Of Botanical Garden Perlis And About Asin-Asin Plant. In person.

7. Thilagamani, S., Shanti, N.," Gaussian and gabor filter approach for object segmentation", Journal of Computing and Information Science in Engineering, 2014, 14(2), 021006, https://doi.org/10.1115/1.4026458

8. Y. Wu, K. Wang, and F. Tao, "Classification of stored-grain insects based on the extend shearlet transform,"Krawtchouk Moment SVM. J. Chin. Cereals Oils Assoc, vol. 30, no. 11, pp. 103_109, 2015.

9. H. Zhang, H. Mao, and D. Qiu, "Feature extraction of image classification on storedgrain insects," Trans. Chin. Soc. Agric. Eng., vol. 25, no. 2, pp. 126_130, 2009.

10. Rhagini, A., Thilagamani, S. ,'Women defence system for detecting interpersonal crimes",International Journal of Advanced Science and Technology, 2020, Volume 29,Issue7S, pp. 1669-1675

11. Zhi-Chun Huang, Patrick P. K. Chan, Wing. W. Y. Ng, Daniel S. Yeung. 2010. Content Based Image Retrieval Using COLOR Moment And Gabor Texture Feature. Proceedings of 9th International Conference On Machine Learning And Cybernetics, Qingdao, 11-14 July. 719-724.

12. K.Deepa, S.Thilagamani, "Segmentation Techniques for Overlapped Latent Fingerprint Matching", International Journal of Innovative Technology and Exploring Engineering (IJITEE), ISSN: 2278-3075, Volume-8 Issue-12, October 2019. DOI: 10.35940/ijitee.L2863.1081219

13. J. Huang, S. R. Kumar, M. Mitra, W. J. Zhu, and R. Zabih. 1997. Image Indexing Using ColorCorrelograms. IEEE Int. Conf. Computer Vision and Pattern Recognition, San Juan, Puerto Rico. 762-768.

14. Jau-Ling Shih \& Ling-Hwei Chen. Color Image Retrieval Based On Primitives OfColor Moments.

15. Santhi, P., Priyanka, T.,Smart India agricultural information reterival system, International Journal of Advanced Science and Technology, 2020, 29(7 Special Issue), pp. 1169-1175

16. Tajane, Vinita, and N. J. Janwe. 2014. Medicinal Plants Disease Identification Using Canny Edge Detection Algorithm, Analysis and CBIR. International Journal ofAdvance Research in Computer Science and Software Engineering. 4(6): 530-536.

17. 2014. Detection And Classification Of Plant Diseases. International Journal of Advance Research in Computer Science and Software Engineering. 2(6): 868-874.

18. M. S. Prasad Babu and B. SrinivasaRao. 2007. Leaves Recognition Using Back Propagation Neural Network Advice For Pest and Disease Control On Crops. IndiaKisan.Net: Expert Advissory System. 
19. Santhi, P., Lavanya, S., Prediction of diabetes using neural networks, International Journal of Advanced Science and Technology, 2020, 29(7 Special Issue), pp. 1160-1168

20. J. Dai, Y. Li, K. He, and J. Sun, "R-FCN: Object detection via region based fully convolutional networks," in Proc. Adv. Neural Inf. Process. Syst., vol. 7, 2016, pp. 379_387.

21. Vijayakumar, P, Pandiaraja, P, Balamurugan, B \& Karuppiah, M 2019, 'A Novel Performance enhancing Task Scheduling Algorithm for Cloud based E-Health Environment', International Journal of E-Health and Medical Communications (IJEHMC), Vol 10,Issue 2,pp 102-117.

22. P. Pandiaraja, N Deepa 2019 ," A Novel Data Privacy-Preserving Protocol for Multi-data Users by using genetic algorithm”, Journal of Soft Computing, Springer, Volume 23 ,Issue 18, Pages 8539-8553

23. P. F. Felzenszwalb, R. B. Girshick, D. McAllester, and D. Ramanan, “'Object detection with discriminatively trained part-based models," IEEE Trans. Pattern Anal. Mach. Intell., vol. 32, no. 9, pp. 1627_1645, Sep. 2010.

24. N Deepa , P. Pandiaraja, 2020 ," Hybrid Context Aware Recommendation System for E-Health Care by merkle hash tree from cloud using evolutionary algorithm”, Journal of Soft Computing, Springer, Volume 24 ,Issue 10, Pages 7149-7161

A. G. Howard, M. Zhu, B. Chen, D. Kalenichenko, W. Wang, T. Weyand, M. Andreetto, and H. Adam, "MobileNets: Efficient convolutional neural networks for mobile vision applications," 2017, arXiv:1704.04861. [Online]. Available: http://arxiv.org/abs/1704.04861

25. T.-Y. Lin, P. Dollar, R. Girshick, K. He, B. Hariharan, and S. Belongie, 'Feature pyramid networks for object detection," in Proc. IEEE Conf. Comput. Vis. Pattern Recognit. (CVPR), Jul. 2017, pp. 2117_2125.

26. N. Bodla, B. Singh, R. Chellappa, and L. S. Davis, “'Soft-NMS_Improving object detection with one line of code," in Proc. IEEE Int. Conf. Comput. Vis. (ICCV), Oct. 2017, pp. 5561_5569.

27. N Deepa , P. Pandiaraja, 2020 , "E health care data privacy preserving efficient file retrieval from the cloud service provider using attribute based file encryption “, Journal of Ambient Intelligence and Humanized Computing, Springer, https://doi.org/10.1007/s12652-020-01911-5

28. M. D. Zeiler and R. Fergus, "Visualizing and understanding convolutional networks," in Proc. Eur. Conf. Comput. Vis., 2014, pp. 818_833.

29. K. Simonyan and A. Zisserman, "Very deep convolutional networks for large-scale image recognition," 2014, arXiv:1409.1556. [Online]. Available: http://arxiv.org/abs/1409.1556

30. K. He, X. Zhang, S. Ren, and J. Sun, “Deep residual learning for image recognition," in Proc. IEEE Conf. Comput. Vis. Pattern Recognit. (CVPR), Jun. 2016,pp. 770_778.

31. R. Girshick, J. Donahue, T. Darrell, and J. Malik, 'Rich feature hierarchies for accurate object detection and semantic segmentation," in Proc. IEEE Conf. Comput. Vis. Pattern Recognit., Jun. 2014, pp. 580_587.

32. K Sumathi, P Pandiaraja 2019," Dynamic alternate buffer switching and congestion control in wireless multimedia sensor networks", Journal of Peer-to-Peer Networking and Applications, Springer, Volume 13,Issue 6,Pages 2001-2010

33. C. L. Zitnick and P. Dollár, “Edge boxes: Locating object proposals from edges," in Proc. Eur. Conf. Comput. Vis., 2014, pp. 391_405.

34. J. Redmon, S. Divvala, R. Girshick, and A. Farhadi, 'You only look once: Unified, real-time object detection," 2015, arXiv:1506.02640. [Online]. Available: http://arxiv.org/abs/1506.02640

35. Jian F, Jayas D S. The ecosystem approach to grain storage. Agricultural Research, vol. 1(2), pp. 148-156, 2012.

36. Neethirajan S, Karunakaran C, Jayas D S, et al. Detection techniques for stored-product insects in grain. Food Control, vol. 18(2), pp. 157-162, 2007.

37. Zayas I Y, Flinn P W. Detection of insects in bulk wheat samples with machine vision, Transactions of the ASAE, vol. 41(3), pp. 883-888, 1998.

38. X. Zhang et al., "Identification of Maize Leaf Diseases Using Improved Deep Convolutional Neural Networks," IEEE Access., vol. 6, pp. 30370- 30377, 2018.

39. C. Xie, R. Wang, J. Zhang, P. Chen, W. Dong, R. Li, T. Chen and H. Chen, "Multi-level learning features for automatic classification of field crop pests," Computers and Electronics in Agriculture., vol. 152, pp. 233$241,2018$.

40. X. Cheng, Y. Zhang, Y. Chen, Y. Wu and Y. Yue, "Pest identification via deep residual learning in complex background," Computers and Electronics in Agriculture, vol. 141, pp. 351-356, 2017.

41. Qiu, D., Zhang, H., Chen, T., et al. . Software design of an intelligent detection system for stored-grain pests based on machine vision. Trans.Chinese Soc. Agric. Mach., vol. 34 (2), pp. 83-85, 2003.

42. Wu, Y., Wang, K., Tao, F., Classification of stored-grain insects based on the Extend Shearlet Transform, Krawtchouk Moment and SVM. J. Chinese Cereals Oils Assoc, vol. 30(11), pp. 103-109, 2015. 